\title{
COMPARATIVE ANALYSIS OF SUSPENDED SAND CONCENTRATION RECORDED WITH DIFFERENT TECHNIQUES IN A RIPPLED BED REGIME
}

\begin{abstract}
Alireza Ahmari ${ }^{1}$, Hocine Oumeraci ${ }^{2}$, Joachim Gruene ${ }^{1}$
Sediment entrainment processes due to the oscillatory flow above rippled and plane sea beds are fundamentally different. Whereas above plane beds the sheet flow or bed load regime dominates and the momentum transfer is primarily caused by turbulent diffusion, above a sea bed covered with long crested vortex ripples the well organised coherent vortex mechanisms induce sediment-laden lee vortices at the ripple crest, which will be detached from the bed ejected into the water column and finally shed when the flow reverses. To investigate the sediment entrainment processes above vortex ripples, a study was carried out in the Large Wave Flume (GWK) aiming firstly to find out the most appropriate measuring technique to determine the suspended sediment concentration both temporarily and spatially. and secondly to analyse the intra-wave sediment entrainment processes around a steep ripple.
\end{abstract}

Keywords: Non-breaking waves; suspended sediment concentration; sediment transport; Acoustic Backscatter Sensor; intra wave processes; vortex ripple

\section{Introduction}

Non-cohesive sediment, which is permanently transported along the world's coast lines by the action of tides and waves, modify over long time periods both the sea water-land boundary and the coastal bathymetry. Due to the fact that the wave related suspended sediment concentration represents the major part of the total sediment load in the near shore zones, a comprehensive measurement analysis of suspended sand concentration transport is strongly needed.

The recently innovative acoustical and optical measuring techniques with a high temporal and spatial accuracy aiming at the experimental investigation of the sediment transport fundamentals are providing unique insights allowing a reliable description of the sediment transport mechanisms and thus an improved prediction of the evolution of the coastlines and coastal bathymetry.

Since the sea bottom is mostly covered with different sedimentary structures like ripples, the study of the sediment entrainment above a rippled sea bottom both temporally and spatially is of a great importance.

The periodic generation of the lee side vortices due to the flow separation at the steep ripple crests, the laden of the generated vortices with sediment particles, detachment of the sand-rich vortices from the ripple crest, their ejection into the water column and finally the shedding of the detached sediment laden vortices under the flow reversal represent the primarily mechanisms of the sediment entrainment from the sea bottom into the water column (Nielsen 1992, Thorne and Hanes 2002, Thorne et al. 2003, Davies and Thorne 2005).

Experiments with movable sand bed were recently carried out in the Large Wave Flume (GWK) of the Coastal Research Centre (FZK) to measure the suspended sediment concentrations (SSC) under regular and irregular waves using comparatively Multi Frequency Acoustic Backscatter Sensors (ABS), Optical Turbidity meter and Transverse Suction System (TSS).

In the first part of the paper a comparative analysis is discussed to show why acoustical transducers represent the most appropriate technique to measure the temporal and spatial suspended sediment concentration movement above different see bed forms, especially over rippled beds, including the simultaneous measurement of the sea bed profile evolution.

In the second part of the paper the process of the intra-wave sediment entrainment in oscillatory flow around a steep ripple is probed using high multi frequency acoustic backscattering technique.

\section{Experimental facilities and instrumentation}

The investigation was performed aiming at the determination of the suspended sediment dynamics within a collaborative European experiments (HYDRALAB-III) and as a part of a BMBF supported project (ModPro), carried out in the Large Wave Flume (GWK) of the Coastal Research Centre (FZK)

\footnotetext{
${ }^{1}$ Department of civil engineering, Leibniz University Hannover, Coastal Research Centre (FZK), Merkurstrasse 11, 30419 Hannover, Germany

${ }^{2}$ Department of Hydromechanics and Coastal Engineering, Technical University of Braunschweig, Leichtweiß-Institute for Hydraulic Engineering and Water Resources, Beethovenstr. 51a, 38106 Braunschweig, Germany
} 
in Hannover (Germany), a joint institution of both universities Hannover and Braunschweig (Ahmari et al. 2008). The flume has an effective length of $300 \mathrm{~m}$, a width of $5 \mathrm{~m}$ and a depth of $7 \mathrm{~m}$.

A sand layer with the thickness of about $0.82 \mathrm{~m}$ was used to represent the sea bottom. The initial water depth above the sandy bottom was about $3.18 \mathrm{~m}$ (Figure 1).

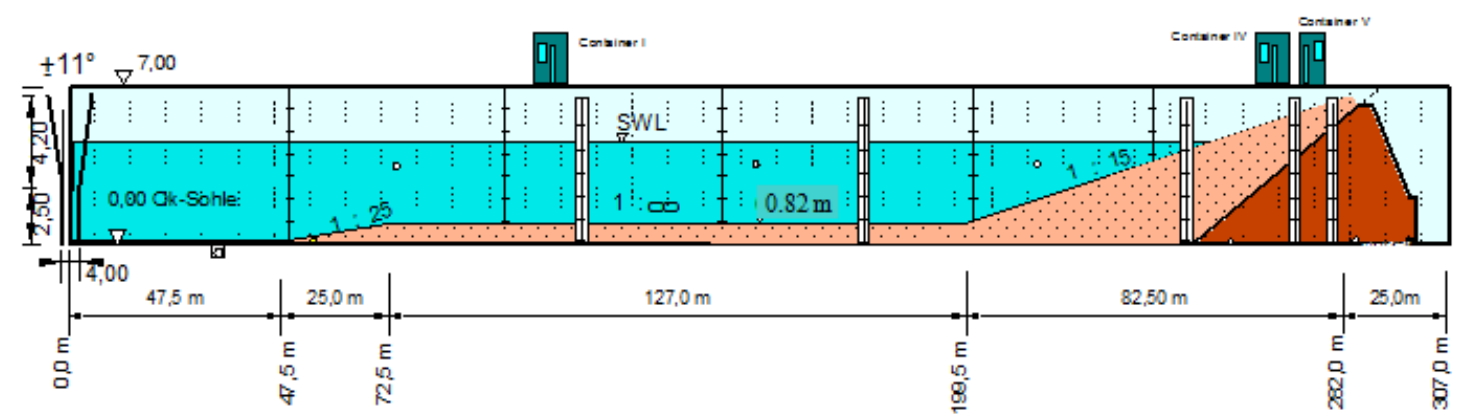

Figure 1: Longitudinal section of the beach profile in the Large Wave Flume (GWK).

The tests were conducted with different wave types: Regular waves ( $H=0.8$ to $1.2 \mathrm{~m}, T=5 \mathrm{~s})$ and irregular waves from JONSWAP-spectra $\left(H_{s}=0.8\right.$ to1.2 $\mathrm{m}$ with $\gamma=1.0$ to 10.0 and $\left.T_{p}=5 \mathrm{~s}\right)$. The two components of the wave velocity $(u$ and $w$ ) were measured using two Electromagnetic Current Meters (ECMs) at two different levels of $0.25 \mathrm{~m}$ and $0.45 \mathrm{~m}$ above the sea bottom. The measurements of the free water surface elevation were performed simultaneously using a wave gauge located in the measuring station (111.45 $\mathrm{m}$ in front of the wave paddle; see Figure 1). Suspended sand concentrations were measured by means of an acoustical device (ABS), an optical device (Turbidity Meter) and a mechanical device (TSS). To verify the acoustic concentrations, the second ABS (ABS-02) was also mounted at $0.81 \mathrm{~m}$ in front of the first one (ABS-01) in the wave propagation direction and at the same height above the sand bottom (see Figure 2).

A sand layer with the thickness of about $0.82 \mathrm{~m}$ laid on the concrete bottom of the GWK-flume represents the sea bed. It is made of well-sorted sand (GWK sand) with a median grain size $D_{50}=0.242 \mathrm{~mm}$ and a non-uniformity factor $U=D_{60} / D_{10}=2.24$ (Figure 1 ).

To provide general background information of the hydrodynamics, three current meters (ECMs) were fitted on the side wall (106.60 $\mathrm{m}$ in front of the wave paddle; see Figure 1) at three different elevations above the sea bed and 22 wave gauges were fitted on the side wall along the entire beach profile to measure simultaneously the free water surface elevation during each test. Figure 2 shows the sandy beach model and the locations of the wave gauges along the Large Wave Flume (GWK).

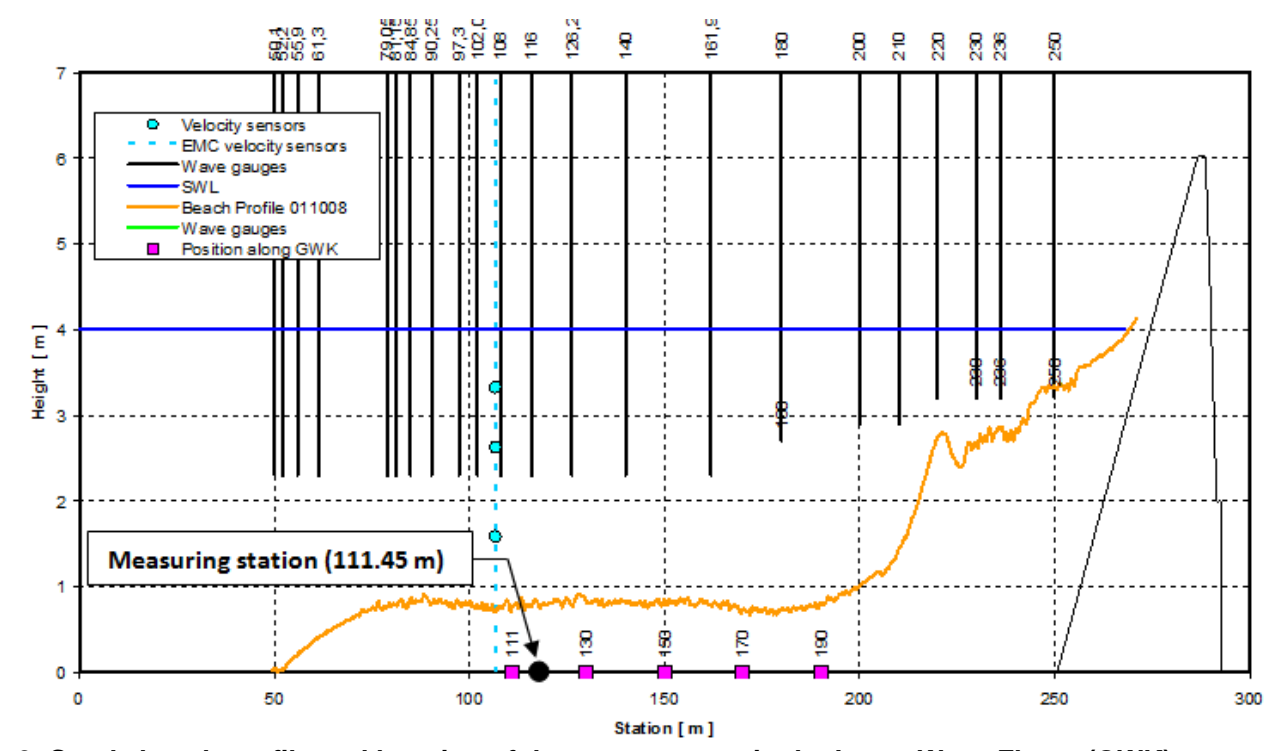

Figure 2: Sandy beach profile and location of the wave gauges in the Large Wave Flume (GWK) 
Figure 3 shows the measurement sensors, which were used to measure the temporal and spatial distribution of the suspended sediment concentrations (SSC) above a moveable sand bed using mechanical (TSS), optical (Optical Turbidity Meter) and acoustical techniques (ABS) including the simultaneous measurements of the water surface elevations and the vertical and horizontal components of the free stream orbital velocity under non breaking regular and irregular waves above the rippled sea bed. Table 1 summaries all measuring devices used in the tests.

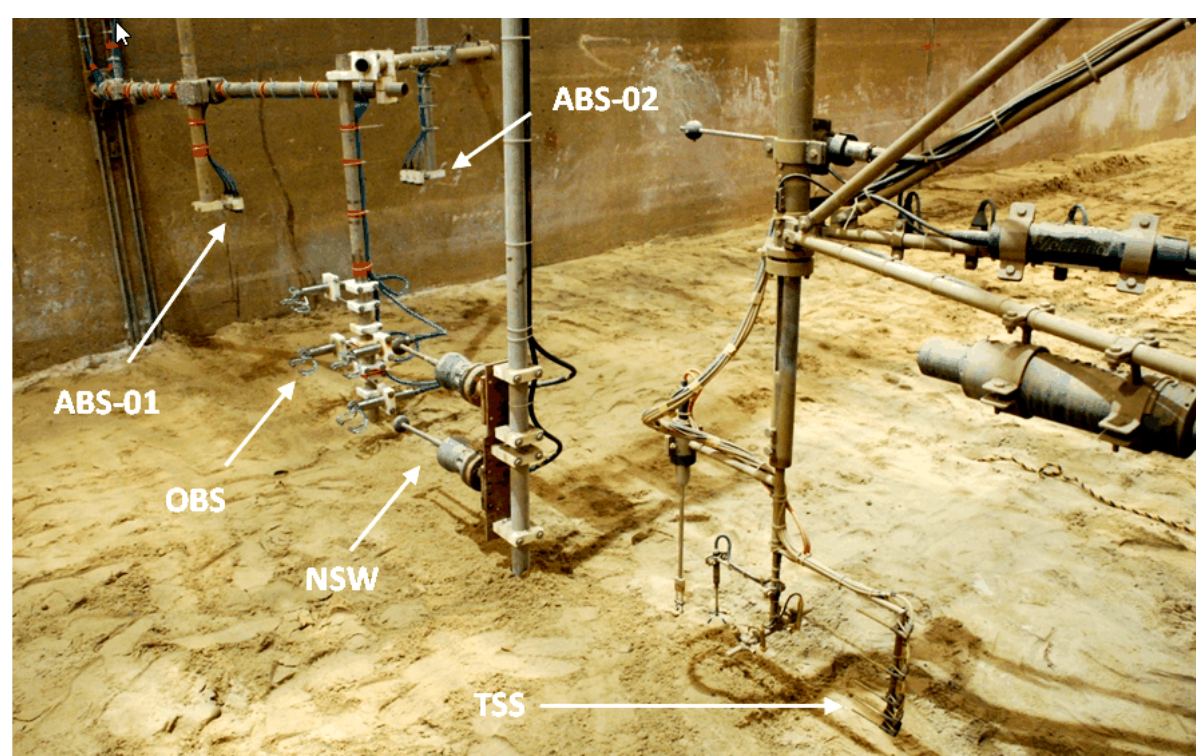

Figure 3: Measuring station and sensors used for experiments in Large Wave Flume (GWK) showing multi frequency Acoustic Backscatter Systems (ABS); Optical point Turbidity meters (OBS); Electromagnetic Current Meters (NSW) and Transverse Suction System (TSS)

Table 1: Instrumentation and sampling rates

\begin{tabular}{|c|c|c|c|}
\hline $\begin{array}{l}\text { Measured } \\
\text { Processes }\end{array}$ & Instrumentation & Measuring location & sampling rate \\
\hline $\begin{array}{l}\text { Free surface } \\
\text { elevation }(\eta)\end{array}$ & Wave gauges (WP) & $\begin{array}{l}21 \text { gauges along the flume } \\
1 \text { gauge at the measurement point (Figure 2) }\end{array}$ & Sampling rate: $40 \mathrm{~Hz}$ \\
\hline $\begin{array}{l}\text { Wave induced } \\
\text { orbital velocity } \\
\qquad(u, w)\end{array}$ & Velocimeter (NSW) & $\begin{array}{l}3 \text { sensors at the wall of the flume } \\
2 \text { sensors at the measurement point near the } \\
\text { bed (Figure } 3 \text { ) }\end{array}$ & Sampling rate: $40 \mathrm{~Hz}$ \\
\hline \multirow{3}{*}{$\begin{array}{l}\text { Suspended } \\
\text { Sand } \\
\text { Concentration } \\
\text { (SSC) }\end{array}$} & $\begin{array}{l}\text { Transverse Suction } \\
\text { System } \\
\text { (TSS) }\end{array}$ & $\begin{array}{l}5 \text { nozzles at the measurement point with } 3 \mathrm{~m} \\
\text { distance from ABS beam at different levels } \\
\text { above the sand bottom (Figure } 3 \text { ) }\end{array}$ & $\begin{array}{l}\text { Extraction of water } \\
\text { and sediment: } 20 \\
\text { mins } \\
\text {-Nozzle speed: } 1.5 \\
\mathrm{~m} / \mathrm{s}\end{array}$ \\
\hline & $\begin{array}{l}\text { Acoustical } \\
\text { Backscatter system } \\
\text { (ABS) }\end{array}$ & $\begin{array}{l}3 \text { transducers with different frequencies (1.0, } \\
2.0 \text { and } 4.0 \mathrm{MHz} \text { ) were mounted } 75 \mathrm{~cm} \text { above } \\
\text { the sand bed (Figure } 3 \text { ) }\end{array}$ & $\begin{array}{l}\text { Number of bins: } 100 \\
\text { Spatial resolution: } \\
0.01 \mathrm{~m} \\
\text { Sampling rate: } 4 \mathrm{~Hz}\end{array}$ \\
\hline & $\begin{array}{l}\text { Optical Turbidity } \\
\text { meter (OBS) }\end{array}$ & $\begin{array}{l}4 \text { transducers were mounted in different } \\
\text { distances from the sand bed at two beams } \\
\text { (Figure 3) }\end{array}$ & Sampling rate: $40 \mathrm{~Hz}$ \\
\hline
\end{tabular}

\section{Measurements of sand concentration in the Large Wave Flume (GWK)}

The results of the measurement and a comparative analysis of the suspended sediment concentration under regular and irregular waves are discussed here. The measurements of the free water surface elevation, two vertical and horizontal components of the wave-induced flow velocity and the suspended concentration events using mechanical, optical and acoustical techniques were conducted in each test.

The tests were performed with different types of waves: Regular waves ( $H=0.8$ to $1.2 \mathrm{~m}, T=5 \mathrm{~s}$ ) and irregular waves from JONSWAP-spectra $\left(H_{s}=0.8\right.$ to1.2m, $T_{p}=5 \mathrm{~s}, \gamma=1.0$ to 10.0). 
The two components of the wave-induced flow velocities ( $u$ and $w$ ) were measured at elevations $0.25 \mathrm{~m}$ and $0.45 \mathrm{~m}$ above the sea bottom. The free water surface elevation was recorded simultaneously by a wave gauge located in the longitudinal axis of the wave flume, at the same distance from the wave paddle as all the other sensors (i.e. $111.45 \mathrm{~m}$ from the wave paddle) (Figure 1).

The analogue signals obtained from the measurement sensors (Current meter, wave gauges and optical turbidity meters ) were sampled by an analogue to digital converter (ADC) with a sampling rate of $40 \mathrm{~Hz}$ and recorded on a hard disk (see Table 1). To ensure the synchronization between the real time measurements (ABS-measurement) and the analogue measurements, the ABS bursts were externally triggered.

The results of the statistical analysis, which quantify the agreement between the acoustical and pump sampled concentrations as well as between the optical and pump sampled measurements of the suspended sediment concentration are shown in Figure 4 and Figure 5 and will be discussed in the following:

Figure 4 shows a comparison between the acoustic and the pump sampled concentrations (Figure 4a, b, c: $C_{\mathrm{ABS}-01}-C_{\mathrm{TSS}}$; Figure 4-d, e, f: $C_{\mathrm{ABS}-02}-C_{\mathrm{TSS}}$ ) conducted at different heights above the sand bed (i.e. at $0.14,0.17,0.21$ and $0.37 \mathrm{~m}$ above the sand bed) beneath regular and irregular waves $\left(H_{s}, H=0.8,1.0,1.2 \mathrm{~m} ; T, T_{p}=5 \mathrm{~s}\right)$. Linear regression on the data gives correlation coefficients $R^{2}=0.9444,0.9679$ and 0.9744 for $C_{\mathrm{ABS}-01}-C_{\mathrm{TSS}}$ plot and $R^{2}=0.8597,0.9645$ and 0.9633 for $C_{\mathrm{ABS}-02}-C_{\mathrm{TSS}}$ plot under $H_{\mathrm{s}}=0.8,1.0$ and $1.2 \mathrm{~m}$ respectively. As can be seen the concentrations lie considerably close to the line $C_{\mathrm{ABS}}-C_{\mathrm{TSS}}$. For ABS-01, the regression gradients were $C_{\mathrm{ABS}-01} / C_{\mathrm{TSS}}=0.9884,0.9944,0.9337$, whereas for ABS-02 they were $C_{\mathrm{ABS}-02} / C_{\mathrm{TSS}}=0.9587,0.10009$, 0.9383 showing that there is no significant scatter from the line ABS=TSS.

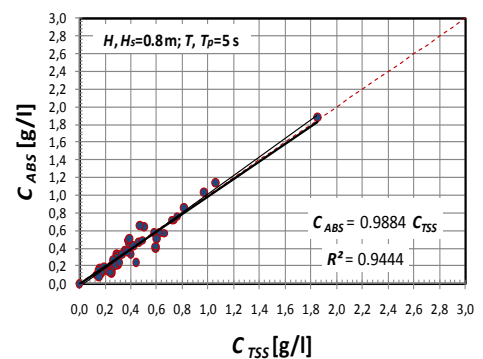

a) ABS-01: $H_{s}, H=0.8 \mathrm{~m}$ Ts, $T=5 \mathrm{~s}$

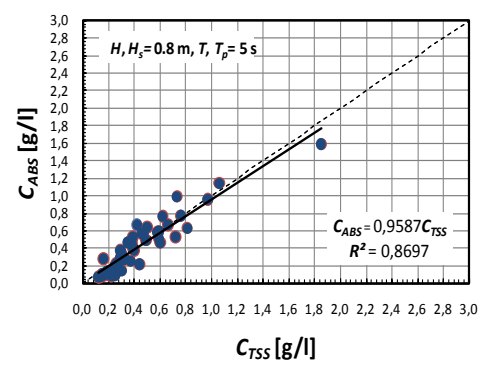

d) ABS-02: $H_{s}, H=0.8 \mathrm{~m}$ Ts, $T=5 \mathrm{~s}$

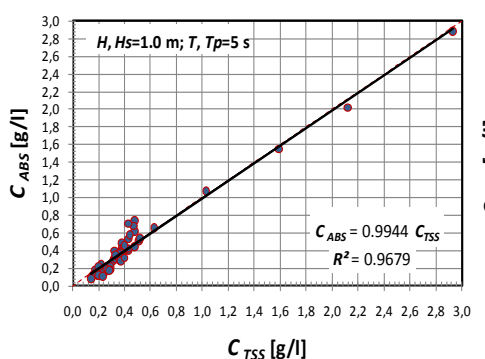

b) ABS-01: $H_{s}, H=1.0 \mathrm{~m} T_{s}, T=5 \mathrm{~s}$

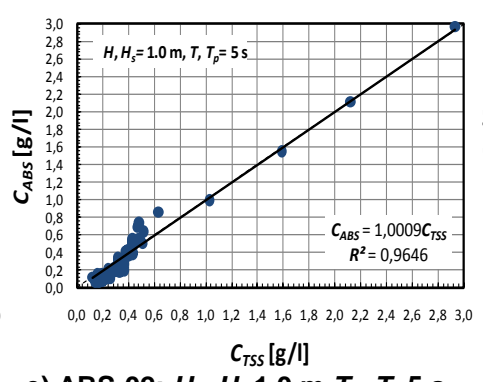

e) ABS-02: $H_{s}, H=1.0 \mathrm{~m} T_{s}, T=5 \mathrm{~s}$

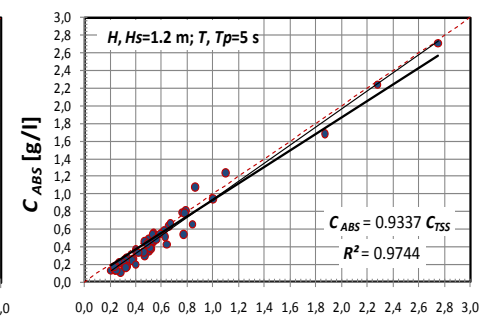

$$
C_{T S S}[\mathrm{~g} / \mathrm{l}]
$$

c) ABS-01: $H_{s}, H=1.2 \mathrm{~m} T_{s}, T=5 \mathrm{~s}$

Figure 4: Regression plots of the mean acoustic concentrations: ABS-01, 02 concentrations against TSS concentrations at $0.14,0.17,0.21$ and $0.37 \mathrm{~m}$ above the sand bed under regular and irregular waves: a,d) Wave height, $H_{s}, H=0.8 \mathrm{~m}$; Wave period, $T_{p}, T=5 \mathrm{~s}$ and peakedness factor $\gamma=1-9.9$. b,e) Wave height, $H_{s}, H=1.0$ $\mathrm{m}$; Wave period, $T_{p}, T=5 \mathrm{~s}$ and $\gamma=1-9.9$. c,f) Wave height, $H_{s}, H=1.2 \mathrm{~m}$; Wave period, $T_{p}, T=5 \mathrm{~s}$ and $\gamma=1-9.9$.

Figure 5 shows a comparison between the concentrations measured by mean of the Optical Turbidity Meters and the pump sampled concentrations conducted at $0.12,0.23,0.32$ and $0.46 \mathrm{~m}$ above the sand bed under regular and irregular waves ( Wave conditions: $H_{s}, H=0.8,1.0,1.2 \mathrm{~m} ; T, T_{p}=5 \mathrm{~s}$; $\gamma=1-9.9)$.

Linear regression on the data gives a correlation coefficient $R^{2}=0.98,0.99$ and 0.9935 for $C_{\mathrm{OBS}^{-}}$ $C_{\mathrm{TSs}}$ plot under $H_{\mathrm{s}}=0.8,1.0$ and $1.2 \mathrm{~m}$ respectively. For optical measurements, the regression gradients 
were $C_{\mathrm{OBS}} / C_{\mathrm{TSS}}=1.12,1.115,1.060$. A good agreement between time averaged TSS- and turbidity meter concentrations ( $C_{\mathrm{TSS}}$ and $C_{\mathrm{OBS}}$ ) for the concentrations range less than $1 \mathrm{~g} / \mathrm{l}$ can be observed.

However, the regression analysis of the concentration results calculated by means of the Turbidity meter shows an overestimation tendency compared to the TSS concentrations.

This discrepancy, which is more significant at higher concentrations, should be due to the larger light beam attenuations in the regions very close to the sea bottom, where the high concentration events occur. This is mostly due to the re-suspension of more fine muddy materials in the water used for the tests in the wave flume.

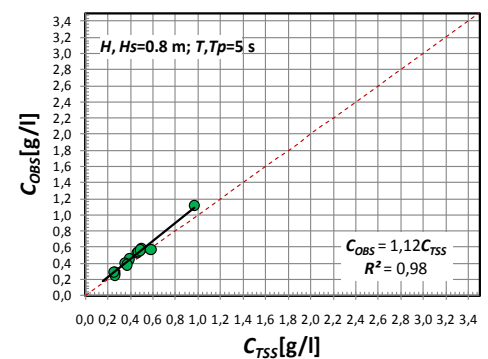

a) ABS-02: $H_{s}, H=0.8 \mathrm{~m} T_{s}, T=5 \mathrm{~s}$

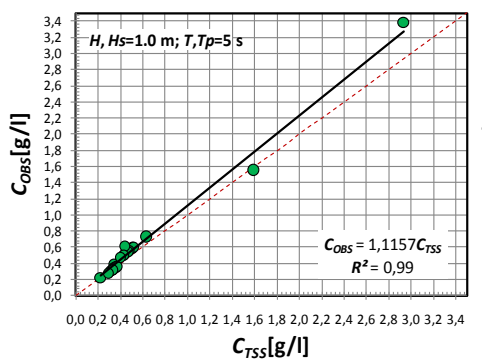

b) ABS-02: $H_{s}, H=1.0 \mathrm{~m} T_{s}, T=5 \mathrm{~s}$

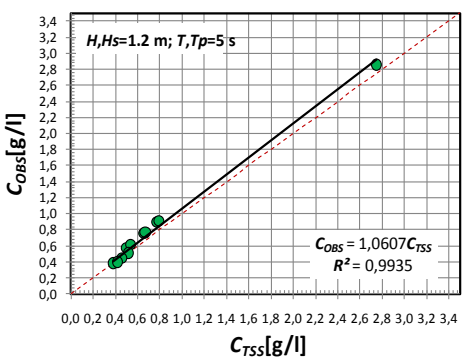

c) ABS-02: $H_{\mathrm{s}}, H=1.2 \mathrm{~m} T_{\mathrm{s}}, T=5 \mathrm{~s}$

Figure 5: Regression plots of the mean concentrations measured by Turbidity meter against pumped sample (TSS) concentrations at $0.12,0.23,0.32$ and $0.46 \mathrm{~m}$ above the sand bed under regular and irregular waves: a) Wave height, $H_{s}, H=0.8 \mathrm{~m}$; Wave period, $T_{p}, T=5 \mathrm{~s}$ and peakedness factor, $\gamma=1-9.9$. b) Wave height, $H_{s}, H=1.0$ $\mathrm{m}$; Wave period, $T_{p}, T=5 \mathrm{~s}$ and $\gamma=1-9.9$ and c) Wave height, $H_{s}, H=1.2 \mathrm{~m}$; Wave period, $T p, T=5 \mathrm{~s}$ and $\gamma=1-9.9$.

\section{Effect of the peak enhancement factor}

To investigate the dependency of the mean suspended sediment concentration on the different JONSWAP-spectra related to the Pierson-Moskowitz spectrum $(\gamma=1)$, experiments have been performed under irregular waves $\left(H_{s}=0.8,1.0\right.$ and $\left.1.2 \mathrm{~m}\right)$ with increasing peakedness factor, $\gamma=1.0-9.9$, by each wave height category.

Figure 6-a, b and c show quantitatively the fluctuations of the time-averaged suspended sand concentrations due to the increase in the peakedness factor, $\gamma$, using ABS measurement data set. The figures show a general decreasing tendency of mean ABS concentrations at different heights above the sea bed as the peak of the wave spectral density grows (i.e. with increasing $\gamma$ ).

Figure 6-d, e and f show the change in the standard deviation of the suspended concentration fluctuations during each time series measured by ABS-01 at $0.14,0.17,0.21$ and $0.37 \mathrm{~m}$ above the sand bed (the horizons of the TSS intake nozzles). Figures show clearly that the fluctuation of the suspended sediment concentrations at the higher concentration levels close to the sea bed are more significant with increasing $\gamma$ than at the lower concentration levels, so that the closer to the sea bed the more significant the effect of the $\gamma$ becomes on the oscillation of the mean suspended concentrations.

Observation of the mean concentration versus peakedness factor, $\gamma$, (Figure 6-a,b,c) for three $H_{s}$-categories $\left(H_{s}=0.8,1.0\right.$ and $\left.1.2 \mathrm{~m}\right)$ with a constant peak period $\left(T_{p}=5 \mathrm{~s}\right)$ under irregular waves show a relative decrease in the mean concentration magnitude with the increasing peakedness factor, which is more significant during the tests with $H_{s}=1.0$ and $1.2 \mathrm{~m}$.

Moreover, within each $H_{s}$-category, a significant change in the concentration magnitude can be seen with an increase in the peakedness factor up to $\gamma=3.3$. With further increase in peakedness factor from $\gamma=3.3$ the mean concentration generally tends to decrease again.

This can be observed more significantly when looking into the change of the standard deviation of the suspended concentration fluctuations plotted against peakedness factor shown in Figure 6- d, e, f.

Here, the oscillations of the standard deviation of the time-averaged suspended concentration up to $\gamma=3.3$ is significant. Further increase in the peak enhancement factor, $\gamma$, causes a general decreasing tendency of the mean suspended sediment concentration in the mean suspended concentration for each $H_{\mathrm{s}}$-category. 


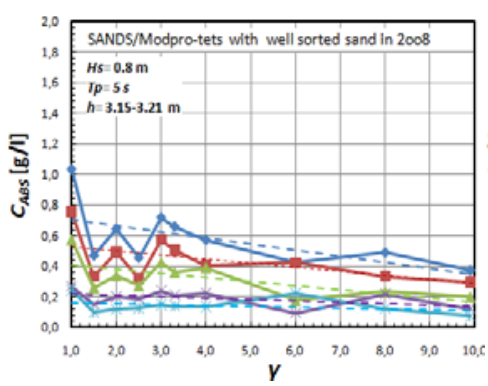

a) $H_{s}=0.8 \mathrm{~m}$

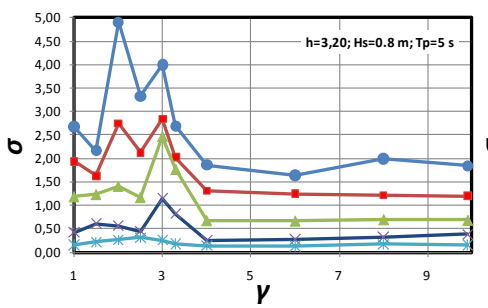

d) $H_{s}=0.8 \mathrm{~m}$

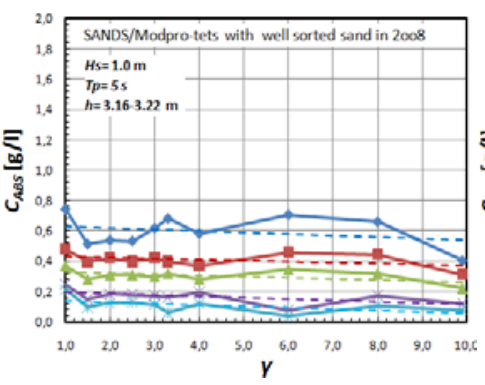

b) $H_{s}=1.0 \mathrm{~m}$

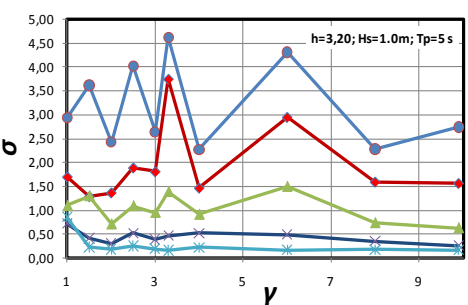

e) $H_{s}=0.8 \mathrm{~m}$

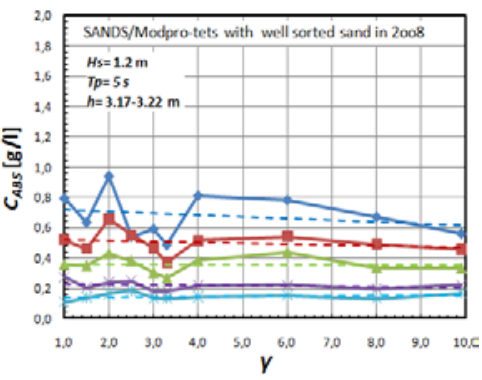

c) $H_{\mathrm{s}}=1.2 \mathrm{~m}$

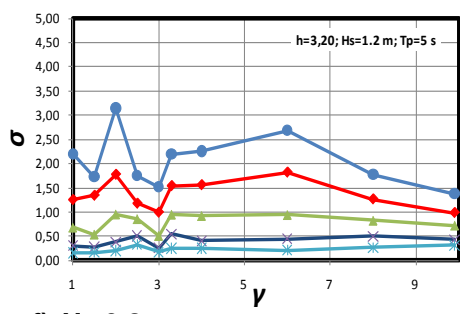

f) $H_{s}=0.8 \mathrm{~m}$

$$
\begin{array}{r}
-14 \mathrm{~cm} \text { above the bottom } \\
-21 \mathrm{~cm} \text { above the bottom } \\
-37 \mathrm{~cm} \text { above the bottom }
\end{array}
$$$$
\leadsto 17 \mathrm{~cm} \text { above the bottom }
$$$$
\div 28 \mathrm{~cm} \text { above the bottom }
$$

Figure 6: $a, b$ and c) Effect of peakedness factor, $y$, on ABS mean concentration at the TSS measuring horizons using the GWK- sand $\left(D_{50}=0.224 \mathrm{~mm}\right)$; $\mathrm{d}$, e and f) Standard deviation of the suspended sediment concentration fluctuations at TSS levels against peakedness factor, $\gamma$, obtained by ABS-0. 8

\section{Sediment entrainment over steep ripples}

In contrast to the plane bed, above rippled bed, the primarily sediment entrainment due to the intra wave flow velocity occurs, when a vortex is formed on the lee side of the ripple due to the flow separation at the ripple crest and laden with sediment particles by rolling on the ripple surface at the time of the positive wave half cycle and detached and advected into the water column at the time of the flow reversals. Therefore, to investigate the sediment entrainment both temporarily and spatially, a simultaneously measurement of the bed profile evolution in the same measuring point is importantly needed, which is best possible using ABS.

Figures 7-a and b show the full migration of two vortex ripples beneath ABS-01 during a test with regular waves (Wave condition: $H=1.2 \mathrm{~m} T=5 \mathrm{~s}$ ). Figure 7-a shows a ripple migrated beneath ABS-01 during $360 \mathrm{~s}$ (Ripple 1), whereas Figure 7-b shows a ripple migration with a duration of $720 \mathrm{~s}$ (Ripple 2).

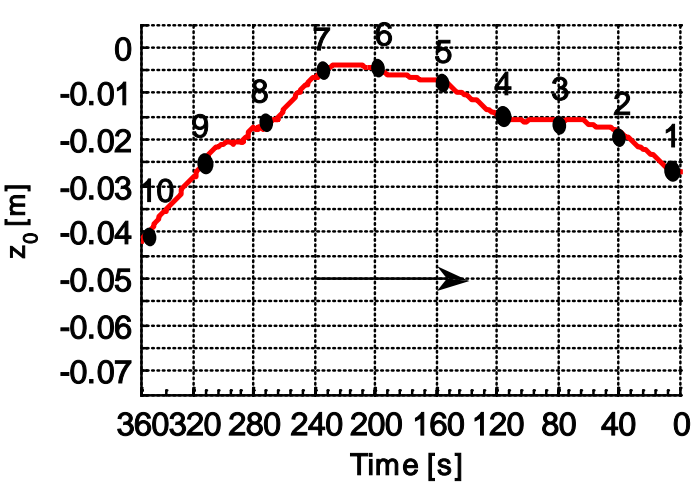

a) Ripple 1

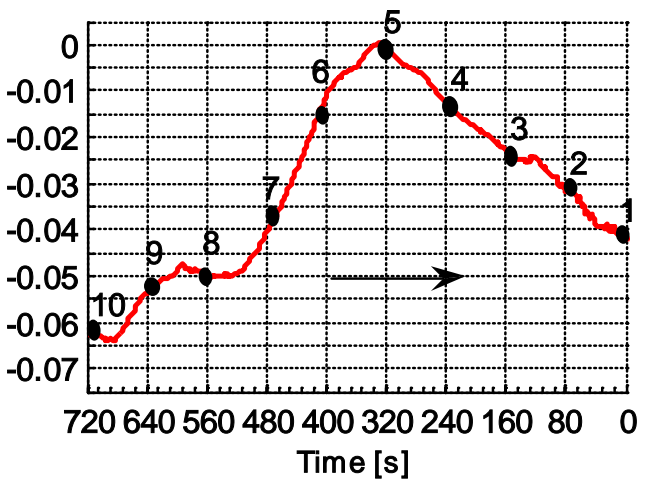

b) Ripple 2

Figure 7: Profile of the fully migrated ripple beneath ABS-01 during the measurement period. The arrows show the direction of the ripple migrations 
The arrows show the direction of the wave propagation, which is the direction of the ripple migration as well (Thorne et al., 2002). The ripples fully migrated beneath the ABS-01 during the course of one test period (Test condition: $H=1.2 \mathrm{~m}, T=5 \mathrm{~s}, h=3.18 \mathrm{~m}$, test duration: $33 \mathrm{~min} ; 400$ waves).

Taking into account that the ripples illustrated in Figures 7-a,b migrated during one test, the assumption can be made that the migration velocity for both ripples was approximately the same. This shows that the ripple 2 (see Figure 7-b) is steeper than the ripple 1 as illustrated in Figure 7-a. To investigate the sand entrainment over both ripples, high resolution patterns of phase-averaged beddependent suspended concentration fields around the ripples within a superimposed wave cycle is presented in the following.

To investigate the concentration pattern more precisely, the sediment fluctuations by coincidence must be avoided. Random fluctuations of flow velocity and bed geometry in the measuring station and in the adjacent regions, can lead to large scatters and attenuations in the instantaneous concentration measurement. To avoid such effects, the velocities in successive wave cycles were superimposed to indicate the repeatability of the regular weakly asymmetrical waves during the observational period. The corresponding time- and ripple-dependent phase-averaged suspended concentrations calculated above both ripples illustrated in Figure 7-a,b for 20 phase angles of the superimposed wave cycle are shown in Figure 8.

Figure 8 compares the phase averaged time- and ripple-dependent concentration peaks calculated with ABS-measurement data set under weakly asymmetric regular waves $(H=1.2 \mathrm{~m}, T=5)$ for two ripples (Ripple 1 and 2) with different forms and sizes (see Figures 7-a and -b) under the same test condition. The panels on the top show the horizontal component of the free stream wave velocity measured with electromagnetic current meter at $0.25 \mathrm{~m}$ above the sea bed. The panels 1to10, in both Figures 8-a and-b, represent the corresponding phase-averaged intra wave concentration results measured by means of the ABS-01 during a wave cycle for ten equally spaced locations on the recorded sand ripples. The ABS measurements were obtained in vertical bins at the heights of $0.01 \mathrm{~m}$. The intra wave concentration panels 1 to 10 illustrated in Figure 8-a and b represent the phaseaveraged concentration peaks, which correspond to numbered locations on the ripple 1 and ripple 2, over 7 and 14 wave periods, respectively, down to a height of $0.01 \mathrm{~m}$ above the ripple crests, when the numbered locations was below the ABS-01(see also Figures 7-a,b).

Concentration panels 1-5 for both ripples, illustrated in Figures 8-a and -b, represent the numbered locations at the on-shore flank of the ripple 1 and ripple 2, whereas the panels 7-10 represent the numbered location on the offshore slope of the ripple 1 and ripple 2 illustrated in Figures 7-a and b.

High sediment entrainment event is observed at the lee slope of both ripples at the time of the maximum positive flow velocity (see concentration panels 1to 5 in Figures 8-a and b). Compared with the concentration pattern 1to 5 over the ripple 1 shown in Figure 7 -a the sediment entrainment and concentration peaks above ripple 2 (Figure 7-b) occurring between $\varphi=0$ and $\pi / 2$, where $\varphi$ is the phase angle, are more significant, which is due to the fact that the ripple 2 is much steeper than the ripple 1. Therefore, the generation of lee vortices over the ripple 2 is stronger.

Looking into the concentration patterns for the locations 1 to 5 above both ripples makes clear that from point 1 to point 5, the concentration peak under maximum positive flow velocity, occurs between $\varphi=0$ and $\pi / 2$, decays in magnitude and suffers a phase lag with increasing distance from the ripple surface, while the concentration clouds under the negative wave half cycle, $\pi / 2<\varphi<3 \pi / 2$, increase.

This well illustrates that the sediment laden vortices generated due to the flow separation at the ripple crest under the positive wave half cycle rolls towards the ripple crests, when the flow reverses. Furthermore, the sharp drop of the concentration peak under the negative wave half cycle is a consequence of the vortex shedding at the time of the flow reversal.

At locations 7 to 10 and 6 to 10 on the off-shore slope of ripple 1 and ripple 2 respectively (see Figures 7-a,b), the similar temporal structure can be observed, where sediment clouds were also developed at the ripple lee sides during the negative velocity maximum, $\pi<\varphi<3 \pi / 2$. However, this second concentration event is weaker than the first one, which is due to the wave asymmetry.

After the phase angle $\varphi=3 \pi / 2$, as the flow start to reverse again, the concentration clouds reduce significantly, which evidence that the vortex generated under the negative wave half cycle is detached and transported in the positive flow direction over the ripple crest.

The high concentration peaks occurred immediately after the negative maximum flow and the second zero crossing point, $\pi<\varphi<3 \pi / 2$, at locations 1 to 5 and 1 to 6 on the on-shore flank of ripple 1 and ripple 2 respectively shown in Figure 8-a and Figure 8-b (see also Figures 7-a,b), are most likely 
associated with the passage of the detached vortices generated at the offshore flank of an adjacent vortex in the down-wave direction during the negative half-cycle of the flow, which was insonified later by means of ABS.

Similarly, the concentration peaks occurred under positive wave half cycle, $0<\varphi<\pi / 2$ and $3 \pi / 2<\varphi<2 \pi$, at locations 6 to 10 and 7 to 10 on the onshore slope of ripple 1 and ripple 2 respectively, shown in Figure 8-a and Figure 8-b (see also Figures 7-a,b), represent probably the insonified vortices generated under the previous negative wave half wave cycle at the lee-slope of the adjacent ripple in the up-wave direction, which are transported during the succeeding positive wave half cycle towards ABS.
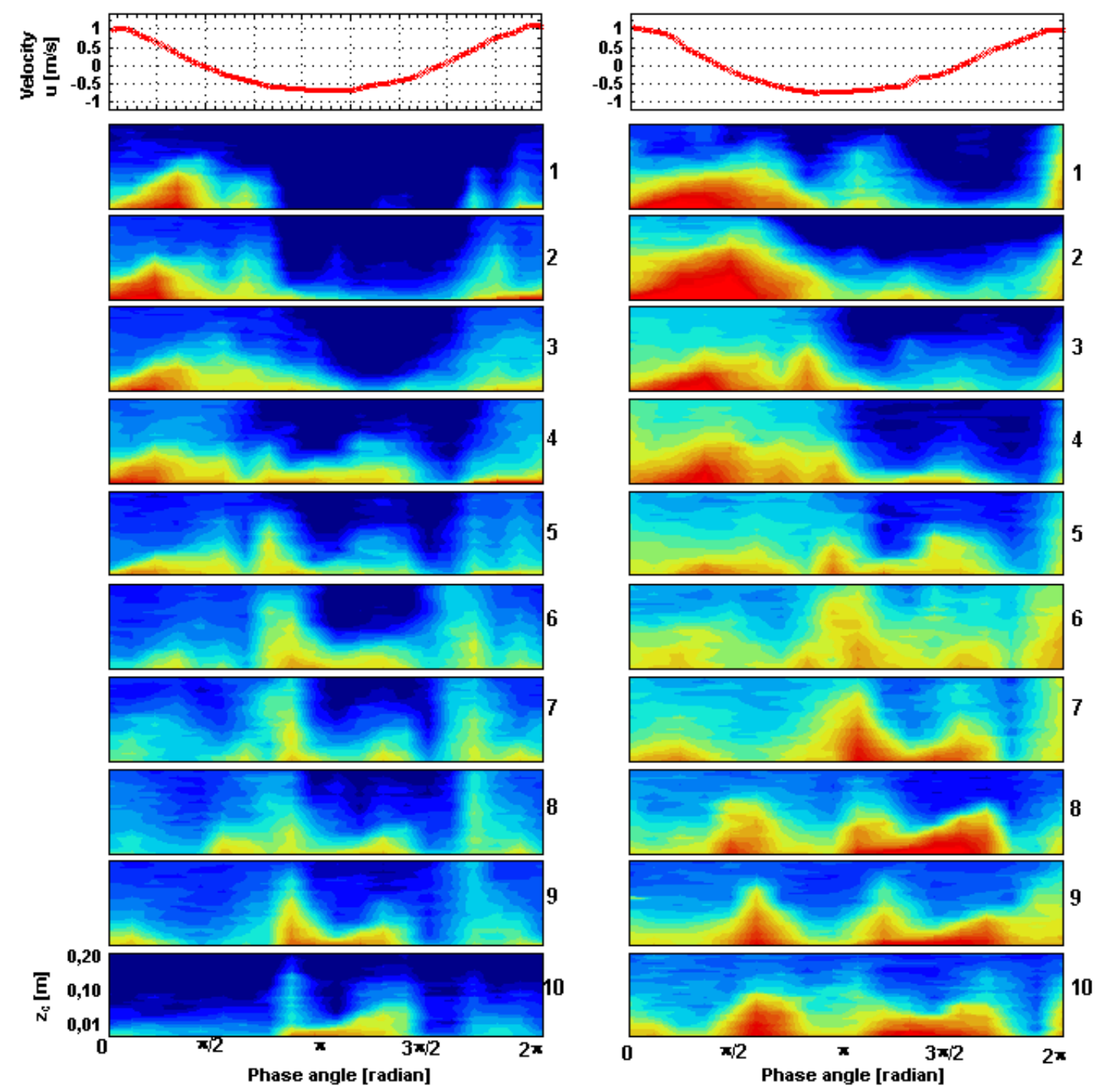

a) SSC above ripple 1

b) SSC above ripple 2

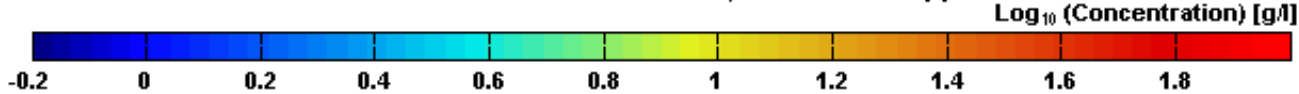

Figure 8: Panels on the top: Intra wave ECM velocity measured at $0.25 \mathrm{~m}$ above the sea bottom. Panels 1 to 10: Ensemble phase-averaged, time and ripple-dependent Suspended Sediment Concentration (SSC) for a test conducted with regular weakly asymmetric waves: $H=1.2 \mathrm{~m} T=5 \mathrm{~s} h=3.18 \mathrm{~m}$. The concentration panels 1 to 10 correspond to numbered locations on ripple 1 and 2 illustrated in Figures 7-a and b. 
Another visualisation of the suspended concentration event around ripples is given in Figure 9-a and $b$, where the phase- and ripple-averaged concentration fields over ripple 1 and 2 shown in Figure 7-a and b are compared.

As can be seen here, four concentration peaks, which are also observed in Figures 8-a and b, can be recognised here again. Looking into the concentration pattern over both ripples shows that the largest sediment ejection, suffering a phase lag, takes place under the maximum flow velocity at $t / T=0.1$ over the ripple 1 (see Figure $9-a$ ) and at $t / T=0.2$ over the ripple 2 (see Figure 9-b) . The second high concentration peak occured at $t / T=0.35$, at the time of the first flow reversal, over the ripple 1 and at $t / T=0.45$ over the ripple 2 , which is somewhat ahead of the first flow reversal. These concentration clouds are probably generated at the lee side of the adjacent ripples, which were transported towards ABS.

The third concentration peak occured at $t / T=0.6$ over the ripple 1 and at $t / T=0.65$ over the ripple 2 (see also Figure 9-a,b). These concentration peaks appear immediately after the maximum negative flow velocity and represent the fact that the passage of the sediment-laden vortices in form of the sediment clouds was observed beneath ABS at the time of the flow reversals.

The fourth concentration peak appears due to the positive flow velocity, which occurs after the second zero crossing point after the second flow reversal $(0.8<t / T<1)$, for both concentration fields shown in panels a and b of Figure 9.

Moreover, the concentration pattern shows that the ripple-averaged concentration within a layer of a thickness of about $15 \mathrm{~cm}$, which approximates three times the ripple height, $\eta$, is highly coherent. This is generally due to the fact that the vortex shedding processes, which normally take place very close to the sea bed, are broken up outside this area and the dominant transport process becomes random turbulence. For the first near bed layer $(z=3 \eta)$ a pure diffusion solution characterised by a ripple height-independent sediment diffusivity coefficient and for the area above this layer the convection-diffusion solution might provide a good representation (Thorne. et al., 2002; 2009).

Furthermore, the concentration event for both ripples evidence that at the time of the second zero crossing point, where the horizontal component of the wave velocity approximates zero and the vertical component of wave velocity is downwards and maximum near the sea bed, the concentration pattern suffers a low point. Consequently, the sand particles remain very close to the ripple surface by the absence of the mobilising forces. This event can clearly be observed by means of both concentration patterns illustrated in Figures 9-a and b over ripple 1 and 2.

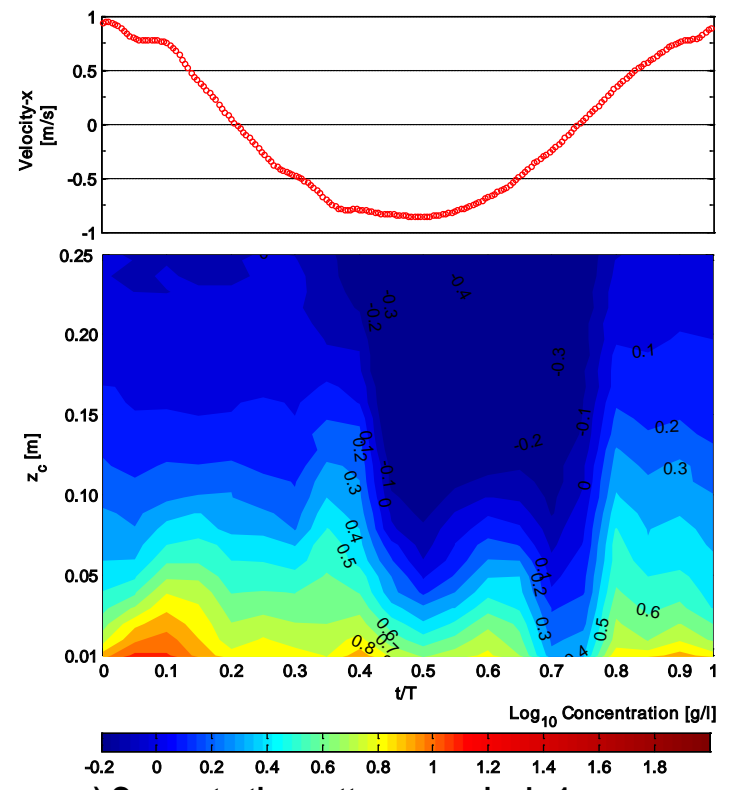

a) Concentration pattern over ripple 1

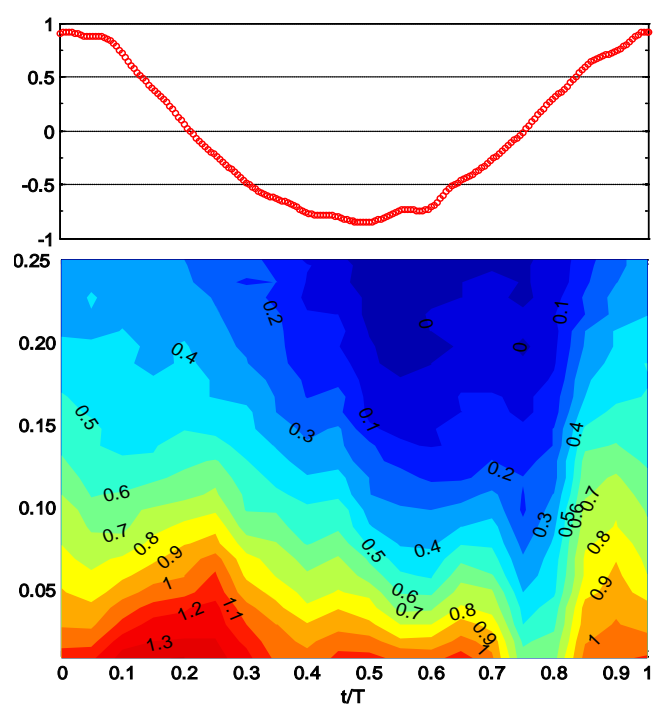

b) Concentration pattern over ripple 1

Figure 9: Time dependent phase- and ripple averaged ABS concentration pattern (Test conditions: $H=1.2 \mathrm{~m}$, $T=5 \mathrm{~s}, h=3.18 \mathrm{~m}$ ). The colors in the contour plots are defined in the color bar as $\log _{10}$ (Concentration). 
Figure 10 shows the time and horizontally averaged vertical concentration profiles conducted by means of the Transverse Suction System (TSS) and calculated from Optical Turbidity Meter and ABSmeasurement data set (ABS-measurements: closed white circles; Turbidity meter measurements: closed red circles and TSS-measurements: closed triangles).

As can be seen here, ABS provides a vertical profile of the suspended concentrations over the sea bed higher up in to the water over a defined vertical operating range up to the transducer head.

The concentrations measured by Optical Turbidity meters (red closed circles) were averaged with duration of about 20 minutes during the test runs. The TSS-measurements (black Triangles) were performed over a time period of about 20 minutes during each test.

A good agreement of the time- and bed-averaged concentration measurement can be observed between the ABS- and the optical concentrations measured by Optical Turbidity Meter and the measured values by means of the Transverse Suction System (TSS).

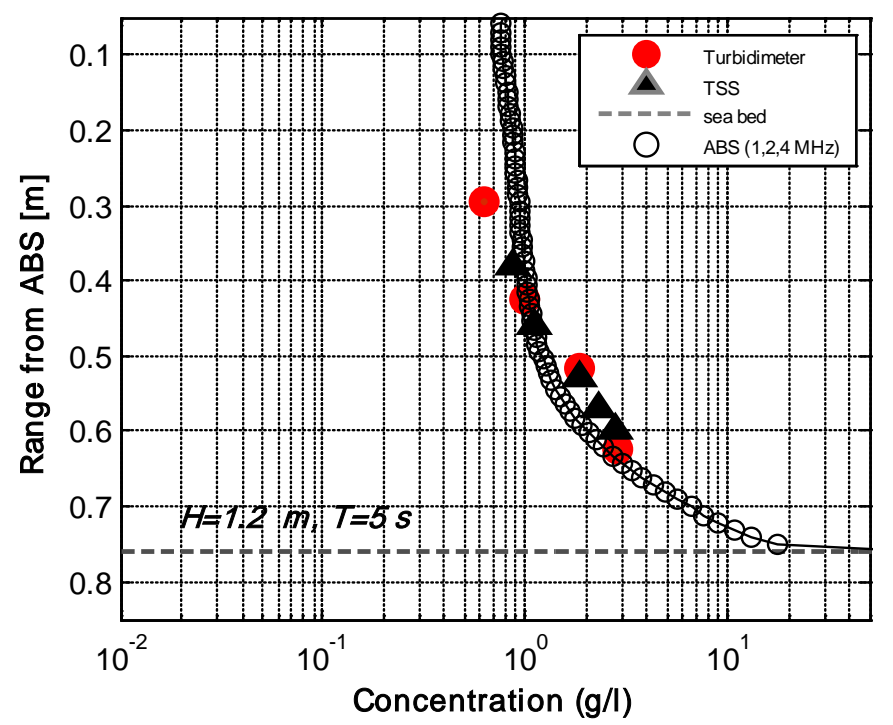

Figure 10 : Time- and bed-averaged concentration: Concentration profiles measured by ABS (1, 2 and $4 \mathrm{MHz}$ transducers) (open white circles); concentration points measured by optical turbidity meter (red closed circles) and by TSS ( closed triangles).

\section{Concluding remarks}

The goal of the presented paper was (i) to comparatively analyse the appropriateness of the most commonly used concentration measurement devices and (ii) to interpret the first results related to the intra wave entrainment sediment processes over two selected steep vortex ripple under weakly asymmetric non breaking regular waves by means of a comparative analysis of concentration events measured by ABS.

The results of the comparative measurement of the suspended sand concentration showed clearly that in most cases there were no significant differences between the time-averaged concentrations measured by means of the ABS and the optical turbidity meter with pump sampled mean concentrations conducted with TSS.

However the optical turbidity meter measurements contain partly a reasonably steady offset concentration by both irregular and regular wave conditions, which is more significant under irregular waves. This is due to the re-suspension of the relatively fine sediments (silt and mud) and should be subtracted from the original time series data. It must also be stressed that, in contrast to the ABS, the turbidity meter did not provide a permanently convincing measurement. This discrepancy should be, in part, due to the larger attenuations at the high concentration levels, which is caused by the resuspension of more fine muddy materials in the water used for the tests in the wave flume.

The fact that ABS is non-intrusive, which provides high- resolution measurement of both suspended sediment concentration and sea bed profile evolution, is a great advantage compared to the 
conventional measuring techniques, which allows a precise interpretation of the processes of sediment entrainment, like vortex formation, vortex shedding and ripple migration.

The well organised temporal and spatial concentration structure provided by means of ABS evidence the fact that the vortex generation mechanisms at the lee side of the steep ripples followed by the shedding of the detached vortices at the time of flow reversal, which represent the primary sediment entrainment processes above steep vortex ripples, are highly coherent.

However, the coherent vortex generation and vortex shedding mechanisms survive just within a near bed area of a height of 2-3 times the ripple height. Outside this area the coherent mechanisms break up and the dominate concentration transport mechanism becomes the random turbulent.

Moreover, the present study showed that in the case of a rippled bed, it is of great importance to consider the phase lags between flow velocities and suspended particles concentration caused by leewake vortices in the rippled-bed regime, which leads to an offshore-directed sediment transport due to the onshore orbital velocities caused by waves.

Furthermore, the characteristics of the ripples, for instance in non-dimensional form such as ripple steepness, $\eta / \lambda$, and relative ripple length, $d_{0} / \lambda$ ( $\eta$ is the ripple height; $\lambda$ the ripple length and $d_{0}$ the orbital diameter), which depend on the strength and the nature of the flow, including steady or tidal currents, waves or a combination of them, are very significant in terms of modeling and predicting of the suspended sediment transport above rippled beds more reliably.

Taking into account that the standard turbulent diffusion models, which are used to predict the near bed time averaged suspended sediment concentration profile, provide an unrealistic description of the intra wave physical processes (Thorne, et al., 2002), the future models of suspended sediment transport in the rippled bed regime must represent the sediment entrainment processes much more accurately, thus highlighting the importance of the high resolution concentration measurement in time and space.

\section{ACKNOWLEDGMENTS}

The work presented in this paper was partly supported by European Community's Sixth Framework Program in the Joint Research Activity SANDS, which is a part of the Integrated Infrastructure Initiative HYDRALAB III, Contract no. 022441 (R113) and by the BMBF supported project ModPro.

\section{REFERENCES}

Ahmari A., Gruene, J., Oumeraci, H. 2008, Large scale laboratory measurement of suspended sediment concentration induced by non-breaking waves with acoustic backscatter technique, Proc. on App. of Phy. Mod., Coastlab’08, Bari, Italy, 93-105.

Nielsen P.1992, Coastal Bottom Boundary Layers and Sediment Transport, Advanced series on Coastal Engineering- Volume 4, World Scientific,

Thorne, P. D., Williams, J.J. and Davies, A.G. 2002, Suspended sediment under waves measured in a large-scale flume facility. Journal of Physical Research. Vol. 107, No. C8, 4.1-4.16.

Thorne, P. D. and Hanes D. M. 2002. A review of the application of acoustics to small scale sediment processes. Continental Shelf Research, 22(4), 603-632.

Thorne, P. D., Davies A. G., Williams J. J. 2003. Measurements of near bed intra-wave sediment entrainment above vortex ripples. Geophys. Res. Lett., Vol. 30, No. 20, 2028, OCE, 2-1 - 2-4.

Thorne, P. D., Davies, A. G. and Bell, Paul S. 2009, Observations and analysis of sediment diffusivity profiles over sandy rippled beds under waves, J. Geophys. Res. Vol. 114 C02023. 1-16.

Davies, Alan G., Thorne, P. D. 2008, Advance in the Study of Moving Sediment and Evolving Seabed, Surv Geophys 29:DOI 10.1007/s10712-008-9039-x. 1-36. 